\title{
Generalized Functionality for Arithmetic Discrete Planes
}

\author{
Valerie Berthé, Christophe Fiorio, and Damien Jamet \\ LIRMM, Université Montpellier II, \\ 161 rue Ada, 34392 Montpellier Cedex 5 - France \\ \{berthe, jamet, fiorio\}@lirmm.fr
}

\begin{abstract}
The discrete plane $\mathfrak{P}(a, b, c, \mu, \omega)$ is the set of points $(x, y, z) \in$ $\mathbb{Z}^{3}$ satisfying $0 \leq a x+b y+c z+\mu<\omega$. In the case $\omega=\max (|a|,|b|,|c|)$, the discrete plane is said naive and is well-known to be functional on a coordinate plane. The aim of our paper is to extend the notion of functionality to a larger family of arithmetic discrete planes by introducing a suitable orthogonal projection direction $(\alpha, \beta, \gamma)$ satisfying $\alpha a+\beta b+\gamma c=\omega$. We then apply this functionality property to the enumeration of some local configurations, that is, the $(m, n)$-cubes such as introduced in VC99.
\end{abstract}

Keywords: digital planes; arithmetic planes; local configurations; functionality of discrete planes.

The discrete plane $\mathfrak{P}(a, b, c, \mu, \omega)$ is the set of integer points $(x, y, z) \in \mathbb{Z}^{3}$ satisfying $0 \leq a x+b y+c z+\mu<\omega$. In the case $\omega=\max (|a|,|b|,|c|)$, the discrete plane is said naive and is well-known to be functional on one of the coordinate planes, that is, for any point of $P$ of this coordinate plane, there exists a unique point in the discrete plane obtained by adding to $P$ a third coordinate. Naive planes have been widely studied, see for instance Rev91, DRR94, DR95, AAS97. VC97, Col02, BB02.

The present paper extends the notion of functionality for naive discrete planes to a larger family of arithmetic discrete planes. For that purpose, instead of projecting on a coordinate space, we introduce a suitable orthogonal projection on a plane along a direction $(\alpha, \beta, \gamma)$, in some sense dual to the normal vector of the discrete plane $\mathfrak{P}(a, b, c, \mu, \omega)$, that is, $\alpha a+\beta b+\gamma c=\omega$, so that the projection of $\mathbb{Z}^{3}$ and the points of the discrete plane are in one-to-one correspondence. One interest of the notion of functionality is that it reduces a threedimensional problem to a two-dimensional one, allowing a better understanding of the combinatorial and geometric properties of discrete planes. We thus apply this functionality property to the enumeration of some local configurations, the $(m, n)$-cubes, for a large family of arithmetic discrete planes, following the approach of [Vui99, BV01.

For clarity issues, we have chosen to work here in a three-dimensional space but all the results and methods presented extend in a natural way to $\mathbb{R}^{n}$, with $n \geq 2$, as well as to arithmetic discrete lines. 


\section{Basic Notions and Arithmetic Discrete Planes}

Let $(a, b, c) \in \mathbb{R}^{3}, \mu \in \mathbb{R}$ and $\omega \in \mathbb{R}_{+}^{\star} ;$ the arithmetic discrete plane $\mathfrak{P}(a, b, c, \mu, \omega)$ is defined as follows:

$$
\mathfrak{P}(a, b, c, \mu, \omega)=\left\{(x, y, z) \in \mathbb{Z}^{3} \mid 0 \leq a x+b y+c z+\mu<\omega\right\} .
$$

Moreover, if $\omega=\max \{|a|,|b|,|c|\}$ (resp. $\omega=|a|+|b|+|c|)$ then $\mathfrak{P}(a, b, c, \mu, \omega)$ is said to be naive (resp. standard).

In the present paper, in order to simplify the notation and to facilitate the generalization of our results to higher dimensions, we use a vector-based representation. Let $\left\{\overrightarrow{e_{1}}, \overrightarrow{e_{2}}, \overrightarrow{e_{3}}\right\}$ be the canonical basis of the $\mathbb{R}$-vector space $\mathbb{R}^{3}$. Let $\vec{v}$ and $\overrightarrow{v^{\prime}}$ be two vectors of $\mathbb{R}^{3}$. The notation $\left(\vec{v}, \overrightarrow{v^{\prime}}\right)$ stands for the usual scalar product in $\mathbb{R}^{3}$. Let $i \in\{1,2,3\}$, we denote by $v_{i}=\left(\vec{v}, \overrightarrow{e_{i}}\right)$ the $i$-th coordinate of $\vec{v}$ related to the basis $\left\{\overrightarrow{e_{1}}, \overrightarrow{e_{2}}, \overrightarrow{e_{3}}\right\}$.

Hence, for any arithmetic discrete plane $\mathfrak{P}$, there exist a vector $\vec{v} \in \mathbb{R}^{3}$ and two real numbers $\mu \in \mathbb{R}$ and $\omega \in \mathbb{R}_{+}^{\star}$ such that

$$
\mathfrak{P}=\left\{\vec{x} \in \mathbb{Z}^{3} \mid 0 \leq(\vec{x}, \vec{v})+\mu<\omega\right\} .
$$

In the sequel of this paper, we denote such a plane by $\mathfrak{P}(\vec{v}, \mu, \omega)$. For a given $\vec{\alpha} \in \mathbb{Z}^{3}$, let $\Pi_{\vec{\alpha}}: \mathbb{R}^{3} \rightarrow\left\{\vec{x} \in \mathbb{R}^{3} \mid(\vec{\alpha}, \vec{x})=0\right\}$ stand for the orthogonal projection map onto the plane $(\vec{\alpha}, \vec{x})=0$. We furthermore use the notation $\pi_{\vec{\alpha}}$ when we consider the restriction of the projection $\Pi_{\vec{\alpha}}$ to a subset of $\mathbb{R}^{3}$, as for instance $\pi_{\vec{\alpha}}: \mathfrak{P} \rightarrow\left\{\vec{x} \in \mathbb{R}^{3} \mid(\vec{\alpha}, \vec{x})=0\right\}$, for a discrete plane $\mathfrak{P}$.

Let us recall a classical property of naive discrete planes having a positive normal vector:

Theorem 1. DRR94 Let $\mathfrak{P}=\mathfrak{P}(\vec{v}, \mu, \omega)$ be a naive discrete plane. If $v_{i}=\omega$, for $i=1,2$ or 3 , then $\mathfrak{P}$ is in bijection with the integer points of the plane $\left(\overrightarrow{e_{i}}, \vec{x}\right)=0$ by the projection map $\Pi_{\overrightarrow{e_{i}}}$, that $i s$, the restriction map $\pi_{\overrightarrow{e_{i}}}: \mathfrak{P} \longrightarrow$ $\Pi_{\overrightarrow{e_{i}}}\left(\mathbb{Z}^{3}\right)$ is a bijection. The plane $\left(\overrightarrow{e_{i}}, \vec{x}\right)=0$ is called a functional plane of $\mathfrak{P}$.

An analogous result holds for standard discrete planes:

Theorem 2. $B V 00$ Let $\mathfrak{P}=\mathfrak{P}(\vec{v}, \mu, \omega)$ be a standard discrete plane. Let $\vec{\alpha}=\overrightarrow{e_{1}}+\overrightarrow{e_{2}}+\overrightarrow{e_{3}}$. Then, the restriction map $\pi_{\alpha}: \mathfrak{P} \longrightarrow \Pi_{-\alpha}\left(\mathbb{Z}^{3}\right)$ is a bijection.

\section{Generalized Functionality}

First, let us notice that in each of the two cases investigated in Theorem 1 1 and 2 the following property holds: let $\mathfrak{P}$ be a naive or a standard discrete plane with normal vector $\vec{v}$ and with thickness $\omega$; then there exists a vector $\vec{\alpha}$ in $\mathbb{Z}^{3}$ such that the restriction map $\pi_{\alpha}: \mathfrak{P} \longrightarrow \Pi_{-\alpha}\left(\mathbb{Z}^{3}\right)$ is a bijection, and $(\vec{\alpha}, \vec{v})=\omega$.

In this section, we extend this property to any discrete plane $\mathfrak{P}(\vec{v}, \mu, \omega)$ whatever its thickness $\omega$ by introducing a dual vector $\vec{\alpha} \in \mathbb{Z}^{3}$ 
such that $(\vec{v}, \vec{\alpha})=\omega$. Furthermore, we improve this result by showing that the projections $\pi_{\vec{\alpha}}$ are the only ones which provide a one-to-one correspondence between the discrete plane $\mathfrak{P}(\vec{v}, \mu, \omega)$ and the projection of $\mathbb{Z}^{3}$; this will then yield a one-to-one correspondence between a discrete plane and a twodimensional lattice.

\subsection{A Bijective Projection for Arithmetic Discrete Planes}

Theorem 3. Let $\mathfrak{P}=\mathfrak{P}(\vec{v}, \mu, \omega)$ be a discrete plane where $\vec{v} \in \mathbb{R}^{3}$ is a nonzero vector, $\mu \in \mathbb{R}$ and $\omega \in \mathbb{R}_{+}^{*}$. Let $\vec{\alpha} \in \mathbb{Z}^{3}$ such that $\operatorname{gcd}\left(\alpha_{1}, \alpha_{2}, \alpha_{3}\right)=1$ and $(\vec{\alpha}, \vec{v}) \neq 0$. Then, $\pi_{\vec{\alpha}}: \mathfrak{P} \longrightarrow \Pi_{\vec{\alpha}}\left(\mathbb{Z}^{3}\right)$ is a bijection if and only if $|(\vec{\alpha}, \vec{v})|=\omega$.

The proof of Theorem 3 first requires a technical lemma:

Lemma 1. Let $\mathfrak{P}=\mathfrak{P}(\vec{v}, \mu, \omega)$ be a discrete plane with $(\vec{v}, \mu, \omega) \in \mathbb{R}^{3} \times \mathbb{R} \times$ $\mathbb{R}_{+}^{*}$.

1. If $\operatorname{dim}_{\mathbb{Q}}\left(v_{1}, v_{2}, v_{3}\right)=1$, then there exists $\left(\overrightarrow{v^{\prime}}, \mu^{\prime}, \omega^{\prime}\right) \in \mathbb{Z}^{3} \times \mathbb{Z} \times \mathbb{N}$ such that $\mathfrak{P}=\mathfrak{P}\left(\overrightarrow{v^{\prime}}, \mu^{\prime}, \omega^{\prime}\right)$ and $\operatorname{gcd}\left(v_{1}^{\prime}, v_{2}^{\prime}, v_{3}^{\prime}\right)=1$.

2. If $\operatorname{dim}_{\mathbb{Q}}\left(v_{1}, v_{2}, v_{3}\right)>1$, then the family $((\vec{x}, \vec{v})+\mu)_{\vec{x} \in \mathfrak{P}}$ is dense in $[0, \omega[$.

Proof. 1. Let us suppose that $\operatorname{dim}_{\mathbb{Q}}\left(v_{1}, v_{2}, v_{3}\right)=1$. Then, there exists $\zeta \in$ $\mathbb{R}_{+}^{\star}$ such that $\left(\zeta v_{1}, \zeta v_{2}, \zeta v_{3}\right) \in \mathbb{Z}^{3}$. Let $\overrightarrow{v^{\prime}}=\zeta \vec{v}, \mu^{\prime}=\lceil-\zeta \mu\rceil$ and $\omega^{\prime}=$ $\lceil\zeta \omega-\zeta \mu\rceil-\lceil-\zeta \mu\rceil$. An easy computation gives $\mathfrak{P}(\vec{v}, \mu, \omega)=\mathfrak{P}\left(\overrightarrow{v^{\prime}}, \mu^{\prime}, \omega^{\prime}\right)$. Finally, according to [AAS97], $\vec{v}$ can be chosen with $\operatorname{gcd}\left(v_{1}, v_{2}, v_{3}\right)=1$.

2. If $\operatorname{dim}_{\mathbb{Q}}\left(v_{1}, v_{2}, v_{3}\right)>1$, then we conclude by the classical following result: the set $\left\{m+n \alpha \mid(m, n) \in \mathbb{Z}^{2}\right\}$ is dense in $\mathbb{R}$ if $\alpha \notin \mathbb{Q}$.

With the hypothesis of Lemma 1 let us observe that $\mathfrak{P}\left(\overrightarrow{v^{\prime}}, \mu^{\prime}, \omega^{\prime}\right)$ is a naive (resp. standard) discrete plane, if so is $\mathfrak{P}(\vec{v}, \mu, \omega)$.

Proof of Theorem 目. We assume w.l.o.g that $(\vec{\alpha}, \vec{v})>0$. Let $\vec{x}=\left(x_{1}, x_{2}, x_{3}\right)$, $\overrightarrow{x^{\prime}}=\left(x_{1}^{\prime}, x_{2}^{\prime}, x_{3}^{\prime}\right) \in \mathbb{Z}^{3} ; \pi_{\vec{\alpha}}(\vec{x})=\pi_{\vec{\alpha}}\left(\overrightarrow{x^{\prime}}\right)$ if and only if there exists $\left(k, k^{\prime}\right) \in \mathbb{Z}^{2}$ such that $k^{\prime}\left(\overrightarrow{x^{\prime}}-\vec{x}\right)=k \vec{\alpha}$. With no loss of generality we can suppose that $\operatorname{gcd}\left(k, k^{\prime}\right)=1$; then, $k^{\prime}$ divides $\operatorname{gcd}\left(\alpha_{1}, \alpha_{2}, \alpha_{3}\right)$ and $\left|k^{\prime}\right|=1$. In other words, $\pi_{\vec{\alpha}}(\vec{x})=\pi_{\vec{\alpha}}\left(\overrightarrow{x^{\prime}}\right)$ if and only if there exists $k \in \mathbb{Z}$ such that $\overrightarrow{x^{\prime}}=\vec{x}+k \vec{\alpha}$. Moreover, $\vec{x}+k \vec{\alpha} \in \mathfrak{P}$ if and only if

$$
\frac{-((\vec{x}, \vec{v})+\mu)}{(\vec{\alpha}, \vec{v})} \leq k<\frac{\omega-((\vec{x}, \vec{v})+\mu)}{(\vec{\alpha}, \vec{v})} .
$$

1) Let us first assume that $(\vec{\alpha}, \vec{v})=\omega$. Then,

$$
\# \llbracket \frac{-((\vec{x}, \vec{v})+\mu)}{(\vec{\alpha}, \vec{v})}, \frac{\omega-((\vec{x}, \vec{v})+\mu)}{(\vec{\alpha}, \vec{v})} \llbracket=1,
$$

and we have proved that $\pi_{\vec{\alpha}}: \mathfrak{P} \longrightarrow \Pi_{\vec{\alpha}}\left(\mathbb{Z}^{3}\right)$ is a bijection. 
2) Conversely, let us assume that $\pi_{\vec{\alpha}}: \mathfrak{P} \longrightarrow \Pi_{\vec{\alpha}}\left(\mathbb{Z}^{3}\right)$ is a bijection.

i. If $\operatorname{dim}_{\mathbb{Q}}\left(v_{1}, v_{2}, v_{3}\right)=1$, then, thanks to Lemma 1, we can suppose that $\vec{v} \in \mathbb{Z}^{3}$, with $\operatorname{gcd}\left(v_{1}, v_{2}, v_{2}\right)=1$, and $(\mu, \omega) \in \mathbb{Z} \times \mathbb{N}^{\star}$. Let $\vec{x} \in \mathbb{Z}^{3}$ such that $(\vec{x}, \vec{v})+\mu=0$. Then $\vec{x} \in \mathfrak{P}$ and $(\vec{x}+\vec{\alpha}, \vec{v})+\mu=(\vec{x}, \vec{v})+$ $(\vec{\alpha}, \vec{v})+\mu=(\vec{\alpha}, \vec{v})>0$. Moreover, $\pi_{\vec{\alpha}}(\vec{x}+\vec{\alpha})=\pi_{\vec{\alpha}}(\vec{x})$. Since $\pi_{\vec{\alpha}}$ is injective then $\vec{x}+\vec{\alpha} \notin \mathfrak{P}$, and hence $(\vec{\alpha}, \vec{v}) \geq \omega$. On the other hand, let $\overrightarrow{x^{\prime}} \in \mathbb{Z}^{3}$ such that $\left(\overrightarrow{x^{\prime}}, \vec{v}\right)+\mu=-1$. Then, $\left(\overrightarrow{x^{\prime}}+\vec{\alpha}, \vec{v}\right)+\mu=$ $\left(\overrightarrow{x^{\prime}}, \vec{v}\right)+(\vec{\alpha}, \vec{v})+\mu=(\vec{\alpha}, \vec{v})-1 \geq 0$. Since $\pi_{\vec{\alpha}}$ is surjective and $(\vec{\alpha}, \vec{v})>0$, then $\vec{x}+\vec{\alpha} \in \mathfrak{P}$, that is, $(\vec{\alpha}, \vec{v})-1<\omega$, or equivalently, $(\vec{\alpha}, \vec{v}) \leq \omega$.

ii. Let us suppose that $\operatorname{dim}_{\mathbb{Q}}\left(v_{1}, v_{2}, v_{3}\right) \geq 2$. Then, each interval $\left[\frac{-((\vec{x}, \vec{v})+\mu)}{(\vec{\alpha}, \vec{v})}, \frac{\omega-((\vec{x}, \vec{v})+\mu)}{(\vec{\alpha}, \vec{v})}[\right.$, with $\vec{x} \in \mathfrak{P}$, contains one and exactly one integer if and only if $(\vec{\alpha}, \vec{v})=\omega$ by Lemma 1 .

Projecting according to $\vec{\alpha}$ corresponds to looking at the plane along a direction parallel to $\vec{\alpha}$. Moreover, Theorem 3 states that, looking at the discrete plane $\mathfrak{P}(\vec{v}, \mu, \omega)$ along this direction, one can see all points of $\mathfrak{P}(\vec{v}, \mu, \omega)$ as if they were on the plane $(\vec{\alpha}, \vec{x})=0$. In Section 2.3 , we show that a natural regular lattice structure emerges from this point of view.

As a generalization of functional planes for naive discrete planes, we define:

Definition 1. Let $\mathfrak{P}=\mathfrak{P}(\vec{v}, \mu, \omega)$ be a discrete plane with $\vec{v} \in \mathbb{R}^{3}$ a non-zero vector, $\mu \in \mathbb{R}$ and $\omega \in \mathbb{R}_{+}^{*}$. Let $\vec{\alpha} \in \mathbb{Z}^{3}$ such that $\pi_{\vec{\alpha}}: \mathfrak{P} \longrightarrow \Pi_{\vec{\alpha}}\left(\mathbb{Z}^{3}\right)$ is a bijection. The plane $(\vec{\alpha}, \vec{v})=0$ is called a (generalized) functional plane of $\mathfrak{P}$.

\subsection{Existence of a Dual Vector}

In the case of an arithmetic discrete plane with normal vector $\vec{v} \in \mathbb{R}^{3}$ and thickness $\omega \in \mathbb{R}_{+}^{\star}$, there is no reason for a vector $\vec{\alpha} \in \mathbb{Z}^{3}$ to exist satisfying $(\vec{\alpha}, \vec{v})=\omega$ (consider the case $\left(v_{1}, v_{2}, v_{3}, \omega\right)$ is $\mathbb{Q}$-free). However, if $\mathfrak{P}(\vec{v}, \mu, \omega)$ is an arithmetic discrete plane with normal vector $\vec{v} \in \mathbb{Z}^{3}$, then, according to Lemma 1, we can suppose that $\omega \in \mathbb{Z}$ and $\operatorname{gcd}\left(v_{1}, v_{2}, v_{3}\right)=1$. We then deduce from Bezout's Lemma that there exists a vector $\vec{\alpha} \in \mathbb{Z}^{3}$ such that $(\vec{\alpha}, \vec{v})=\omega$. Let us prove now that $\vec{\alpha} \in \mathbb{Z}^{3}$ can be chosen such that $\operatorname{gcd}\left(\alpha_{1}, \alpha_{2}, \alpha_{3}\right)=1$.

Theorem 4. Let $\mathfrak{P}(\vec{v}, \mu, \omega)$ be an arithmetic discrete plane with $(\vec{v}, \mu, \omega) \in$ $\mathbb{Z}^{3} \times \mathbb{Z} \times \mathbb{Z}_{+}^{\star}$ and $\operatorname{gcd}\left(v_{1}, v_{2}, v_{3}\right)=1$. There exists $\vec{\alpha} \in \mathbb{Z}^{3}$ such that $(\vec{\alpha}, \vec{v})=\omega$ and $\operatorname{gcd}\left(\alpha_{1}, \alpha_{2}, \alpha_{3}\right)=1$. In other words, there exists $\vec{\alpha} \in \mathbb{Z}^{3}$ such that $\pi_{\vec{\alpha}}$ : $\mathfrak{P}(\vec{v}, \mu, \omega) \longrightarrow \Pi_{\vec{\alpha}}\left(\mathbb{Z}^{3}\right)$ is a bijection.

Proof. Let $\vec{\beta} \in \mathbb{Z}^{3}$ such that $(\vec{\beta}, \vec{v})=1$. Then, $(\omega \vec{\beta}, \vec{v})=\omega$. Let $\vec{u} \in\{\vec{x} \in$ $\left.\mathbb{Z}^{3} \mid(\vec{x}, \vec{v})=0\right\}$, let $d=\operatorname{gcd}\left(u_{1}, u_{2}, u_{3}\right)$ and let $\vec{\alpha}=\omega \vec{\beta}+d^{-1} \vec{u}$. Then, an easy computation gives $(\vec{\alpha}, \vec{v})=\omega$ and $\operatorname{gcd}\left(\alpha_{1}, \alpha_{2}, \alpha_{3}\right)=1$. We end the proof by applying Theorem 3 .

We have illustrated Theorem 4 in Figure 1 in the case of a discrete line for a better visualisation of the situation. 


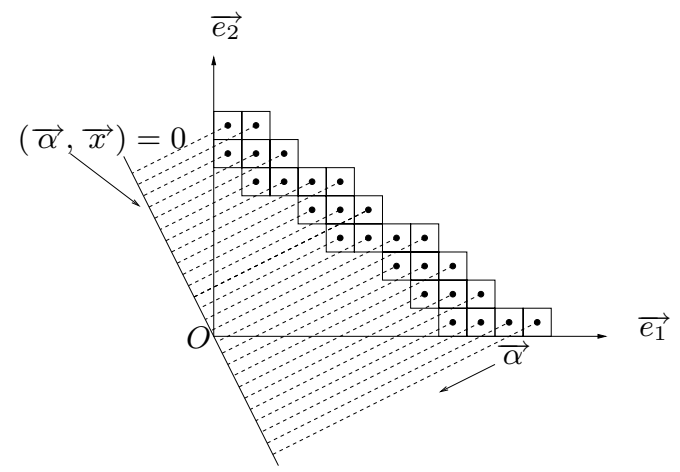

Fig. 1. Generalized functionality: the orthogonal projection of the discrete line $0 \leq$ $7 x_{1}+10 x_{2}+\mu<24$ onto the line $2 x+y=0$

\subsection{Functional Regular Lattice Associated to an Arithmetic Discrete Plane}

Let us see now how any arithmetic discrete plane $\mathfrak{P}$ can be recoded in a functional way on a regular two-dimensional lattice, despite its three-dimensional structure.

Let $\mathfrak{P}=\mathfrak{P}(\vec{v}, \mu, \omega)$ be an arithmetic discrete plane. Let $\vec{\alpha} \in \mathbb{Z}^{3}$ such that $\operatorname{gcd}\left(\alpha_{1}, \alpha_{2}, \alpha_{3}\right)=1$ and $(\vec{\alpha}, \vec{v})=\omega$ (in case $(\vec{v}, \mu, \omega) \in \mathbb{Z}^{3} \times \mathbb{Z} \times \mathbb{N}^{\star}$, and $\operatorname{gcd}\left(v_{1}, v_{2}, v_{3}\right)=1$, the existence of such a vector $\vec{\alpha}$ comes from Theorem (4). One of the coefficients $\alpha_{i}$, for $i \in\{1,2,3\}$ being non-zero, we assume in this section that $\alpha_{3} \neq 0$ with no loss of generality.

First, let us notice that since $\Pi_{\vec{\alpha}}(\vec{\alpha})=\overrightarrow{0}$, then, for all $\vec{x} \in \mathbb{Z}^{3}$,

$$
\Pi_{\vec{\alpha}}\left(\vec{e}_{3}\right)=-\frac{\alpha_{1}}{\alpha_{3}} \Pi_{\vec{\alpha}}\left(\overrightarrow{e_{1}}\right)-\frac{\alpha_{2}}{\alpha_{3}} \Pi_{\vec{\alpha}}\left(\overrightarrow{e_{2}}\right) .
$$

Then, for all $\vec{x} \in \mathbb{Z}^{3}$,

$$
\begin{aligned}
\Pi_{\vec{\alpha}}(\vec{x}) & =x_{1} \Pi_{\vec{\alpha}}\left(\overrightarrow{e_{1}}\right)+x_{2} \Pi_{\vec{\alpha}}\left(\overrightarrow{e_{2}}\right)+x_{3} \Pi_{\vec{\alpha}}\left(\overrightarrow{e_{3}}\right) \\
& =\left(\frac{\alpha_{3} x_{1}-\alpha_{1} x_{3}}{\alpha_{3}}\right) \Pi_{\vec{\alpha}}\left(\overrightarrow{e_{1}}\right)+\left(\frac{\alpha_{3} x_{2}-\alpha_{2} x_{3}}{\alpha_{3}}\right) \Pi_{\vec{\alpha}}\left(\overrightarrow{e_{2}}\right)
\end{aligned}
$$

and

$$
\begin{aligned}
& \Pi_{\vec{\alpha}}: \mathbb{R}^{3} \longrightarrow \quad\left\{\vec{x} \in \mathbb{R}^{3} \mid(\vec{\alpha}, \vec{x})=0\right\} \\
& \vec{x} \mapsto \frac{\alpha_{3} x_{1}-\alpha_{1} x_{3}}{\operatorname{gcd}\left(\alpha_{1}, \alpha_{3}\right)} \overrightarrow{f_{1}}+\frac{\alpha_{3} x_{2}-\alpha_{2} x_{3}}{\operatorname{gcd}\left(\alpha_{2}, \alpha_{3}\right)} \overrightarrow{f_{2}},
\end{aligned}
$$

with

$$
\overrightarrow{f_{1}}=\frac{\operatorname{gcd}\left(\alpha_{1}, \alpha_{3}\right)}{\alpha_{3}} \Pi_{\vec{\alpha}}\left(\overrightarrow{e_{1}}\right) \text { and } \overrightarrow{f_{2}}=\frac{\operatorname{gcd}\left(\alpha_{2}, \alpha_{3}\right)}{\alpha_{3}} \Pi_{\vec{\alpha}}\left(\overrightarrow{e_{2}}\right) .
$$

We thus deduce that $\Gamma_{\vec{\alpha}}=\Pi_{\vec{\alpha}}\left(\mathbb{Z}^{3}\right)=\Pi_{\vec{\alpha}}(\mathfrak{P})$ is a sub-lattice of the twodimensional lattice $\mathbb{Z} \overrightarrow{f_{1}}+\mathbb{Z} \overrightarrow{f_{2}}$. The lattice $\Gamma_{\vec{\alpha}}$ is called a functional lattice of $\mathfrak{P}$. This generalizes the concept of functionality defined for naive discrete planes as a projection onto the integer points of one of the coordinate planes. 


\section{From a Functional Lattice to the Associated Discrete Plane}

Let $\mathfrak{P}=\mathfrak{P}(\vec{v}, \mu, \omega)$ be an arithmetic discrete plane and $\Gamma_{\vec{\alpha}}$ be a functional lattice of $\mathfrak{P}$ (see Section 2.3). A natural question is: "given an element $\vec{y} \in \Gamma_{\vec{\alpha}}$, how can we recover the unique vector $\vec{x} \in \mathfrak{P}$ such that $\pi_{\vec{\alpha}}(\vec{x})=\vec{y}$ ? "In the following, we investigate this question for the classical classes of arithmetic discrete planes, namely the naive, the standard [Rev91, DRR94, DR95] and the graceful ones BB99, BB02.

\subsection{Generalized Functionality for a Particular Class of Discrete Planes}

Let $\mathfrak{P}(\vec{v}, \mu, \omega)$ be an arithmetic discrete plane and let $\vec{\alpha} \in \mathbb{Z}^{3}$ such that $(\vec{\alpha}, \vec{v})=\omega$. In this section, we assume that there exists $i \in\{1,2,3\}$ such that $\alpha_{i}=1$. This condition includes the set of naive, standard and graceful arithmetic planes (see Section [3.2). Let us thus suppose that $\alpha_{3}=1$. In this case, let us notice that $\Gamma_{\vec{\alpha}}=\mathbb{Z} \overrightarrow{f_{1}}+\mathbb{Z} \overrightarrow{f_{2}}$.

Let $\vec{y} \in \Gamma_{\vec{\alpha}}$. From now on, if no confusion is possible with the representation of $\vec{y}$ related to the basis $\left\{\overrightarrow{e_{1}}, \overrightarrow{e_{2}}, \overrightarrow{e_{3}}\right\}$, we will denote $\left(y_{1}, y_{2}\right)$ the unique pair of integers such that $\vec{y}=y_{1} \overrightarrow{f_{1}}+y_{2} \overrightarrow{f_{2}}$.

Let $\vec{x} \in \mathfrak{P}$ and let $\vec{y}=\pi_{\vec{\alpha}}(\vec{x}) \in \Gamma_{\vec{\alpha}}$. According to (11), one has $x_{1}=$ $y_{1}+\alpha_{1} x_{3}$ and $x_{2}=y_{2}+\alpha_{2} x_{3}$. Hence, $(\vec{x}, \vec{v})+\mu=y_{1} v_{1}+y_{2} v_{2}+x_{3}\left(\alpha_{1} v_{1}+\right.$ $\left.\alpha_{2} v_{2}+v_{3}\right)+\mu$ and

$$
0 \leq(\vec{x}, \vec{v})+\mu=v_{1} y_{1}+v_{2} y_{2}+x_{3} \omega+\mu<\omega .
$$

Thus, given any $\vec{y} \in \mathbb{Z}^{2}$, we can easily recover the unique vector $\vec{x} \in \mathfrak{P}$ such that $\pi_{\vec{\alpha}}(\vec{x})=\vec{y}$. Indeed, let us first note that (2) yields an explicit formula for the height $x_{3}$ of the points of $\mathfrak{P}$, that is, $x_{3}=-\left\lfloor\frac{v_{1} y_{1}+v_{2} y_{2}+\mu}{\omega}\right\rfloor$. Let us call $H_{\mathfrak{P}, \vec{\alpha}}: \Gamma_{\vec{\alpha}} \longrightarrow \mathbb{Z}$ the function which to any point $y_{1} \overrightarrow{f_{1}}+y_{2} \overrightarrow{f_{2}} \in \Gamma_{\vec{\alpha}}$ associates the height $x_{3}$ of the corresponding point $\vec{x} \in \mathfrak{P}$, that is, the unique point $\vec{x} \in \mathfrak{P}$ such that $\pi_{\vec{\alpha}}(\vec{x})=\vec{y}$ :

$$
H_{\mathfrak{P}, \vec{\alpha}}: \vec{y} \mapsto-\left\lfloor\frac{v_{1} y_{1}+v_{2} y_{2}+\mu}{\omega}\right\rfloor .
$$

One thus obtains:

Proposition 1. If $\alpha_{3}=1$, then the function $\pi_{\vec{\alpha}}^{-1}: \Gamma_{\vec{\alpha}} \longrightarrow \mathfrak{P}$ is defined by, for all $\vec{y} \in \Gamma_{\vec{\alpha}}:$

$$
\pi_{\vec{\alpha}}^{-1}(\vec{y})={ }^{t}\left(\begin{array}{c}
y_{1} \\
y_{2} \\
0
\end{array}\right)+H_{\mathfrak{P}, \vec{\alpha}}\left(y_{1}, y_{2}\right){ }^{t}\left(\begin{array}{c}
\alpha_{1} \\
\alpha_{2} \\
1
\end{array}\right) .
$$




\subsection{Classical Examples}

Let us suppose that $\vec{v} \in \mathbb{N}^{3}$, and $v_{3}=\max \left\{v_{1}, v_{2}, v_{3}\right\}$. If $\mathfrak{P}$ is a naive or a standard discrete plane, then we can suppose $\alpha_{3}=1$, since $v_{i} \geq 0$ for $i \in\{1,2,3\}$. In the special case of naive discrete planes, we recover the already known formula:

Corollary 1. If $\mathfrak{P}$ is a naive discrete plane, then $\vec{\alpha}=\overrightarrow{e_{3}}$, for all $\vec{x} \in \mathfrak{P}$, $\pi_{\vec{\alpha}}(\vec{x})=x_{1} \overrightarrow{e_{1}}+x_{2} \overrightarrow{e_{2}}$ and for all $\vec{y} \in \Gamma_{\vec{\alpha}}$,

$$
\pi_{\overrightarrow{e_{3}}}^{-1}(\vec{y})=y_{1} \overrightarrow{e_{1}}+y_{2} \overrightarrow{e_{2}}-\left\lfloor\frac{v_{1} y_{1}+v_{2} y_{2}+\mu}{v_{3}}\right\rfloor \overrightarrow{e_{3}} .
$$

Concerning the case of the standard discrete planes, we obtain, as a direct consequence of Proposition 1

Corollary 2. If $\mathfrak{P}$ is a standard discrete plane, then $\vec{\alpha}=\overrightarrow{e_{1}}+\overrightarrow{e_{2}}+\overrightarrow{e_{3}}$, for all $\vec{x} \in \mathfrak{P}, \pi_{\vec{\alpha}}(\vec{x})=\left(x_{1}-x_{3}\right) \overrightarrow{e_{1}}+\left(x_{2}-x_{3}\right) \overrightarrow{e_{2}}$, and for all $\vec{y} \in \Gamma_{\vec{\alpha}}$,

$$
\pi_{\overline{e 3}}^{-1}(\vec{y})=\left(\begin{array}{c}
y_{1} \\
y_{2} \\
0
\end{array}\right)-\left\lfloor\frac{v_{1} y_{1}+v_{2} y_{2}+\mu}{v_{1}+v_{2}+v_{3}}\right\rfloor^{t}\left(\begin{array}{l}
1 \\
1 \\
1
\end{array}\right) .
$$

Let us suppose now that $\mathfrak{P}=\mathfrak{P}(\vec{v}, \mu, \omega)$ is a graceful plane, that is, $0 \leq v_{1}$, $\leq$ $v_{2} \leq v_{3}$ and $\omega=\max \left(v_{1}+v_{2}, v_{3}\right)$. If $v_{1}+v_{2} \leq v_{3}$, then $\mathfrak{P}$ is a naive discrete plane and this case has already been studied. Let us then assume that $\omega=v_{1}+v_{2}$. Let $\vec{\alpha}=\overrightarrow{e_{1}}+\overrightarrow{e_{2}}$. Then, for all $\vec{x} \in \mathfrak{P}, \pi_{\vec{\alpha}}(\vec{x})=\left(x_{1}-x_{2}\right) \overrightarrow{e_{1}}+x_{3} \overrightarrow{e_{3}}$.

Up to a permutation on the set $\left\{\alpha_{1}, \alpha_{2}, \alpha_{3}\right\}$, we recover the following from Proposition 1

Proposition 2. If $\mathfrak{P}$ is a graceful plane. Then $\vec{\alpha}=\overrightarrow{e_{1}}+\overrightarrow{e_{2}}$ and the function $\pi_{\vec{\alpha}}^{-1}: \Gamma_{\vec{\alpha}} \longrightarrow \mathfrak{P}$ is defined by, for all $\vec{y} \in \Gamma_{\vec{\alpha}}$,

$$
\pi_{\vec{\alpha}}^{-1}(\vec{y})={ }^{t}\left(\begin{array}{c}
0 \\
y_{1} \\
y_{2}
\end{array}\right)+\left\lceil\frac{v_{2} y_{1}-v_{3} y_{2}+\mu}{v_{1}+v_{2}}\right\rceil^{t}\left(\begin{array}{l}
1 \\
1 \\
0
\end{array}\right) .
$$

\section{Plane Partitions and Local Configurations}

The aim of this section is to apply the previous results to the study of $(m, n)$ cubes and local configurations, generalizing the study performed for naive planes in [VC97, Sch97, Gér99, VC99, Col02]. For the sake of consistency, we call them here $\vec{m}$-cubes rather than $(m, n)$-cubes.

Let $\mathfrak{P}=\mathfrak{P}(\vec{v}, \mu, \omega)$ be an arithmetic discrete plane and let $\vec{\alpha} \in \mathbb{Z}^{3}$ such that $\operatorname{gcd}\left(\alpha_{1}, \alpha_{2}, \alpha_{3}\right)=1$ and $(\vec{\alpha}, \vec{v})=\omega\left(\right.$ recall that if $\vec{v} \in \mathbb{Z}^{3}$ and $\operatorname{gcd}\left(v_{1}, v_{2}, v_{3}\right)=$ 1 , then the existence of $\vec{\alpha}$ is ensured by Theorem 4 ). Let us assume furthermore that $\alpha_{3}=1$. 

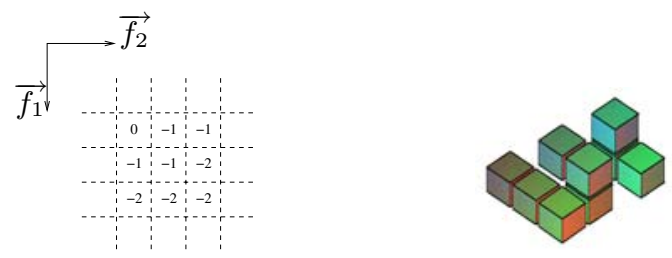

Fig. 2. From left to right: a local configuration of the discrete plane $\mathfrak{P}\left(4 \overrightarrow{e_{1}}+2 \overrightarrow{e_{2}}+\right.$ $\left.5 \overrightarrow{e_{3}}, 0,7\right)$ and its corresponding preimage by $\pi_{\overrightarrow{e_{2}}+\overrightarrow{e_{3}}}^{-1}$

Let $\vec{m} \in\left(\mathbb{N}^{\star}\right)^{2}$ be given. By $\vec{m}$-cube, we mean a local configuration in the discrete plane that can be observed thanks to $\pi_{\vec{\alpha}}$ through an $\vec{m}$-window in the projection lattice $\Gamma_{\vec{\alpha}}$. More precisely,

Definition 2. Let $\vec{m} \in\left(\mathbb{N}^{\star}\right)^{2}$. The $\vec{m}$-cube $\mathcal{C}(\vec{y}, \vec{m})$, with $\vec{y} \in \Gamma_{\vec{\alpha}}$, is defined as the following subset of $\mathfrak{P}$ :

$$
\mathcal{C}(\vec{y}, \vec{m})=\left\{\pi_{\vec{\alpha}}^{-1}(\vec{y}+\vec{i}), \vec{i} \in \llbracket 0, m_{1} \llbracket \times \llbracket 0, m_{2} \llbracket\right\} .
$$

In order to enumerate the different types of $\vec{m}$-cubes that occur in $\mathfrak{P}$, we represent them as local configurations as follows.

Definition 3. The $\vec{m}$-local configuration $L C(\vec{y}, \vec{m})$, with $\vec{y} \in \mathbb{Z}^{2}$ and $m \in$ $\left(\mathbb{N}^{\star}\right)^{2}$, is defined as follows:

$$
L C(\vec{y}, m)=\left[H_{\mathfrak{P}, \vec{\alpha}}(\vec{z})-H_{\mathfrak{P}, \vec{\alpha}}(\vec{y})\right]_{\vec{z} \in \llbracket 0, m_{1}-1\left[\overrightarrow{f_{1}}+\llbracket 0, m_{2}-1 \llbracket \overrightarrow{f_{2}}\right.} .
$$

We say that $\vec{y}$ is an index of occurrence of the local configuration $\operatorname{LC}(\vec{y}, \vec{m})$.

Let us note that a local configuration is a plane partition.

Example 1. For instance, let us consider the arithmetic discrete plane $\mathfrak{P}=$ $\mathfrak{P}(\vec{v}, \mu, \omega)$ with $\vec{v}=4 \overrightarrow{e_{1}}+2 \overrightarrow{e_{2}}+5 \overrightarrow{e_{3}}, \mu=0$ and $\omega=9$. Let $\vec{\alpha}=\overrightarrow{e_{1}}+\overrightarrow{e_{3}}$. We illustrate the local configuration $L C\left(\overrightarrow{f_{1}}+\overrightarrow{f_{2}}, 3\left(\overrightarrow{e_{1}}+\overrightarrow{e_{2}}\right)\right)$ of $\mathfrak{P}$ and its preimage by $\pi_{\vec{\alpha}}^{-1}$ in Fig. 2 .

We follow here the approach developed in Vui99. For a naive discrete plane $\mathfrak{P}$, it is well known that, given two points $\vec{x}$ and $\overrightarrow{x^{\prime}}$ of $\mathfrak{P}$ such that their projections by $\pi_{\vec{\alpha}}$ are 4-connected in the functional plane, then $\left|x_{3}-x_{3}^{\prime}\right| \leq 1$. In other words, the difference between the height of $\vec{x}$ and $\vec{x}$ is at most 1 . A quite unexpected fact is that this property holds for any arithmetic discrete plane with $\alpha_{3}=1$. More precisely, it is easy to see that, for all $\vec{y} \in \Gamma, H_{\mathfrak{P}, \vec{\alpha}}\left(\vec{y}+\overrightarrow{f_{1}}\right)-$ $\left.H_{\mathfrak{P}, \vec{\alpha}}(\vec{y})\right)$ takes only two values, namely $-\left\lfloor\frac{v_{1}}{\omega}\right\rfloor$ and $-\left\lfloor\frac{v_{1}}{\omega}\right\rfloor-1$. In the same way, $\left.H_{\mathfrak{P}, \vec{\alpha}}\left(\vec{y}+\overrightarrow{f_{2}}\right)-H_{\mathfrak{P}, \vec{\alpha}}(\vec{y})\right)$ takes only the values $-\left\lfloor\frac{v_{2}}{\omega}\right\rfloor$ and $-\left\lfloor\frac{v_{2}}{\omega}\right\rfloor-1$. 
In each case, one of these values is odd, whereas the other one is even; we define $E_{h}$ and $O_{h}$ to be respectively the even and the odd value taken by $-\left\lfloor\frac{v_{1}}{\omega}\right\rfloor$ and $-\left\lfloor\frac{v_{1}}{\omega}\right\rfloor-1$; we similarly define $E_{v}$ and $O_{v}$. It is now natural to introduce the following two-dimensional sequence:

$$
U=\left(U_{\vec{y}}\right)_{\vec{y} \in \Gamma_{\vec{\alpha}}}=\left(H_{\mathfrak{P}, \vec{\alpha}}(\vec{y}) \quad \bmod 2\right)_{\vec{y} \in \Gamma_{\vec{\alpha}}} \in\{0,1\}^{\mathbb{Z}^{2}} .
$$

By definition, it is easily seen that the sequence $U$ satisfies:

$$
\forall \vec{y} \in \Gamma_{\vec{\alpha}}, U_{\vec{y}}=0 \text { if and only if }-\frac{y_{1} v_{1}+y_{2} v_{2}+\mu}{\omega} \bmod 2 \in[0,1[.
$$

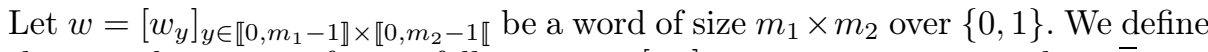

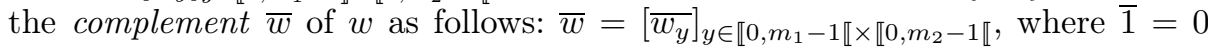
and $\overline{0}=1$. Let us recall [Vui99, BV00 that the set of factors of the sequence $U$ is stable under complementation. We thus introduce the following equivalence relation:

$$
v \sim w \text { if and only if } v \in\{w, \bar{w}\} .
$$

We have the following theorem, inspired by [Vui99]:

Theorem 5. There is a natural bijection between the equivalence classes of the relation $\sim$ of the factors of the sequence $U$ and the $\vec{m}$-local configurations of $\mathfrak{P}$.

Proof. Consider the local configuration $L=L C(\vec{y}, \vec{m})$; we can associate to it the $m_{1} \times m_{2}$ word

$$
[L(\vec{z}) \bmod 2]_{\vec{z} \in \llbracket 0, m_{1}-1 \llbracket \overrightarrow{f_{1}}+\llbracket 0, m_{2}-1 \llbracket \overrightarrow{f_{2}}},
$$

that we denote for short $L \bmod 2$. If $H_{\mathfrak{P}, \vec{\alpha}}(\vec{y})$ is even, then $L \bmod 2$ is a factor of the two-dimensional sequence $U$; otherwise, $H_{\mathfrak{P}, \vec{\alpha}}(\vec{y})$ is odd and $\overline{L \bmod 2}$ is a factor of $U$ and so is $L \bmod 2$, by stability of the set of factors of $U$ by complementation.

Conversely, let us show how we can canonically reconstruct a $\vec{m}$-local configuration, with $\vec{m} \in\left(\mathbb{N}^{\star}\right)^{2}$, from a given $m_{1} \times m_{2}$-factor $w$ of the two-dimensional sequence $U$. Let us first assume that $w_{\overrightarrow{0}}=0$. We define a plane partition $H=[H(\vec{z})]_{\vec{z} \in \llbracket 0, m_{1}-1 \rrbracket \overrightarrow{f_{1}}+\llbracket 0, m_{2}-1 \rrbracket \overrightarrow{f_{2}}}$ by induction as follows: we set $H(\overrightarrow{0})=0$; let $\vec{z} \in \llbracket 0, m_{1}-1 \rrbracket \overrightarrow{f_{1}}+\llbracket 0, m_{2}-1 \rrbracket \overrightarrow{f_{2}}$ be a non-zero vector. If $w_{\vec{z}+\overrightarrow{f_{1}}}=w_{\vec{z}}$, then we set $H\left(\vec{z}+\overrightarrow{f_{1}}\right)=H(\vec{z})+E_{h}$. Otherwise, we set $H\left(\vec{z}+\overrightarrow{f_{1}}\right)=H(\vec{z})+O_{h}$. Similarly, if $w_{\vec{z}+\overrightarrow{f_{2}}}=w_{\vec{z}}$, then we set $H\left(\vec{z}+\overrightarrow{f_{2}}\right)=H(\vec{z})+E_{v}$. Otherwise, we set $H\left(\vec{z}+\overrightarrow{f_{2}}\right)=H(\vec{z})+O_{v}$.

The plane partition $H$ is a local configuration of $\mathfrak{P}$; indeed, if $w$ occurs at index $\vec{y}$ in $U$, then $H=L C(\vec{y}, \vec{m})$ and $w=(H \bmod 2)$ since $H(\vec{y})$ is even (we have $w_{\overrightarrow{0}}=0$ ). Now, if $w_{\overrightarrow{0}}=1$, we apply the same reconstruction process to $\bar{w}$. We recover again a local configuration $L C(\vec{y}, \vec{m})$ such that $\bar{w}=(L C(\vec{y}, \vec{m})$ $\bmod 2)$. 
One deduces, in particular, from Theorem 5 that any local configuration of the discrete plane $\mathfrak{P}$ occurs at least twice: once at an index $\vec{y}$ with $H(\vec{y})$ even and second, at an index $\vec{y}$ such that $H\left(\vec{y}^{\prime}\right)$ is even.

Let us now investigate the enumeration of $\vec{m}$-cubes occuring in a given arithmetic plane. The number of $(3,3)$-cubes included in a given naive arithmetic discrete plane has been proved to be at most 9 in [VC97]. More generally, in Rev95, Gér99, the authors proved that, given a naive arithmetic discrete plane $\mathfrak{P}, \mathfrak{P}$ contains at most $m_{1} m_{2} \vec{m}$-cubes. In the following theorem, we show that this property also holds for $\vec{m}$-local configurations in an arithmetic discrete plane $\mathfrak{P}(\vec{v}, \mu, \omega)$, which is non-necessarily naive.

Theorem 6. Let $\mathfrak{P}=\mathfrak{P}(\vec{v}, \mu, \omega)$ be a discrete plane, $\vec{\alpha} \in \mathbb{Z}^{3}$ such that $(\vec{\alpha}, \vec{v})=\omega$ and $\alpha_{3}=1$, and let $\vec{m} \in\left(\mathbb{N}^{\star}\right)^{2}$. Then, $\mathfrak{P}$ contains at most $m_{1} m_{2}$ $\vec{m}$-local configurations.

Proof. According to [Vui99, BV00, the factors of size $m_{1} \times m_{2}$ of the sequence $U$ are in one-to-one correspondence with the intervals of $\mathbb{R} / 2 \mathbb{Z}$ of extremal points $-\frac{i_{1} v_{1}+i_{2} v_{2}}{\omega}$ and $-\frac{i_{1} v_{1}+i_{2} v_{2}}{\omega}+1$ with $\left(i_{1}, i_{2}\right) \in \llbracket 0, m_{1}-1 \rrbracket \times \llbracket 0, m_{2}-1 \rrbracket$. There are at most $2 m_{1} m_{2}$ such points and the result follows from Theorem 5 .

\section{Conclusion and Perspectives}

The aim of the present work was to introduce suitable tools generalizing the classical ones used in the study of arithmetic discrete planes. We have exhibited a generalized functionality for arithmetic discrete planes $\mathfrak{P}(\vec{v}, \mu, \omega)$ and proved that, as soon as $|(\vec{\alpha}, \vec{v})|=w$ and $\operatorname{gcd}\left(\alpha_{1}, \alpha_{2}, \alpha_{3}\right)=1$, there is a one-to-one correspondence between $\mathfrak{P}$ and a two-dimensional lattice $\Gamma_{\alpha}$. Thanks to these results, we have shown for various classes of arithmetic discrete planes, how to recover $\vec{x} \in \mathfrak{P}$ in correspondence with any $\vec{y} \in \Gamma_{\alpha}$. We also have investigated plane partitions and local configurations and extended the well-known result on the number of $(m, n)$-configurations in a naive plane, that is, there are at most $m n$ such configurations.

This approach offers new perspectives to investigate further general properties of arithmetic discrete planes of any thickness. In particular, we plan to use it to generate arbitrarily large parts of discrete planes via symbolic substitutions following ABS04, to recover the corresponding Farey tesselation as well as the symmetry properties of $\vec{m}$-local configurations of a discrete plane [VC99], and finally as a new approach to the recognition problem of discrete planes [FST96, FP99, VC00].

\section{Acknowledgements}

We would like to thank Fabrice Philippe for a careful reading of this paper. 


\section{References}

[AAS97] Éric Andres, Raj Acharya, and Claudio Sibata. The Discrete Analytical Hyperplanes. Graph. Models Image Process., 59(5):302-309, 1997.

[ABS04] Pierre Arnoux, Valérie Berthé, and Anne Siegel. Two-dimensional Iterated Morphisms and Discrete Planes. Theoret. Comput. Sci., 319:145-176, 2004.

[BB99] Valentin E. Brimkov and Reneta P. Barneva. Graceful Planes and Thin Tunnel-Free Meshes. In DGCI, 8th International Conference, volume 1568 of $L N C S$, pages 53-64, 1999.

[BB02] Valentin E. Brimkov and Reneta P. Barneva. Graceful Planes and Lines. Theoret. Comput. Sci., 283:151-170, 2002.

[BV00] Valérie Berthé and Laurent Vuillon. Tilings and Rotations on the Torus: A Two-Dimensional Generalization of Sturmian Sequences. Discrete Math., 223:27-53, 2000.

[BV01] Valérie Berthé and Laurent Vuillon. Palindromes and Two-Dimensional Sturmian Sequences. J. Autom. Lang. Comb., 6(2):121-138, 2001.

[Col02] Marie Andrée Jacob-Da Col. About Local Configurations in Arithmetic Planes. Theoret. Comput. Sci., 283:183-201, 2002.

[DR95] Isabelle Debled-Renesson. Reconnaissance des Droites et Plans Discrets. Thèse de doctorat, Université Louis Pasteur, Strasbourg, France, 1995.

[DRR94] Isabelle Debled-Renesson and Jean-Pierre Reveillès. A New Approach to Digital Planes. In Vision geometry III, Proc. SPIE, volume 2356, Boston, USA, 1994.

[FP99] Jean Françon and Laurent Papier. Polyhedrization of the boundary of a voxel object. In DGCI, 8th International Conference, volume 1568 of LNCS, pages 425-434, 1999.

[FST96] Jean Françon, Jean-Maurice Schramm, and Mohamed Tajine. Recognizing Arithmetic Straight Lines and Planes. In DGCI, 6th International Workshop, LNCS, pages 141-150, 1996.

[Gér99] Yan Gérard. Local Configurations of Digital Hyperplanes. In DGCI, 8th International Conference, volume 1568, pages 65-75, 1999.

[Rev91] Jean-Pierre Reveillès. Calcul en Nombres Entiers et Algorithmique. Thèse d'état, Université Louis Pasteur, Strasbourg, France, 1991.

[Rev95] Jean-Pierre Reveillès. Combinatorial Pieces in Digital Lines and Planes. In Vision geometry IV, Proc. SPIE, 2573, volume 2573, pages 23-24, San Diego, CA, 1995.

[Sch97] Jean-Maurice Schramm. Coplanar Tricubes. In DGCI, 7th International Workshop, volume 1347 of LNCS, pages 87-98, 1997.

[VC97] Joëlle Vittone and Jean-Marc Chassery. Coexistence of Tricubes in Digital Naive Plane. In DGCI, 7th International Workshop, volume 1347 of LNCS, pages 99-110, 1997.

[VC99] Joëlle Vittone and Jean-Marc Chassery. (n,m)-cubes and Farey Nets for Naive Planes Understanding. In DGCI, 8th International Conference, volume 1568 of $L N C S$, pages 76-87, 1999.

[VC00] Joëlle Vittone and Jean-Marc Chassery. Recognition of Digital Naive Planes and Polyhedrization. In DGCI, 9th International Conference, volume 1953 of $L N C S$, pages 296-307. IAPR, 2000.

[Vui99] Laurent Vuillon. Local Configurations in a Discrete Plane. Bull. Belgian Math. Soc., 6:625-636, 1999. 